\title{
CSF phosphorylated neurofilament subunit NF-H (pNF-H) levels are biomarkers of Spinal Cord Injury
}

\author{
St.M. Iencean ${ }^{1}$, Didona Ungureanu ${ }^{1}$, Al. Tascu ${ }^{2}$, B. Costachescu ${ }^{1}$, \\ A.St. Iencean ${ }^{3}$, I. Poeata ${ }^{1}$ \\ 1“Grigore T. Popa" University of Medicine and Pharmacy Iasi \\ 2"Carol Davila" University of Medicine and Pharmacy Bucharest \\ ${ }^{3}$ Emergency Hospital "Prof. dr. N. Oblu" Iasi
}

\section{Abstract}

Several studies showed that the phosphorylated form of the neurofilament subunit NF-H (pNF-H) are related to neuronal injuries and its detection provide information about the presence and degree of neuronal loss. Neurofilaments are three subunits, namely NF-L, NF-M and NF-H. The phosphorylated neurofilament subunit NF-H (pNF-H) is present into serum and CSF in significant amounts following neuronal injury and may be detected. The pNF-H can be a biomarker of the neuronal injuries and its detection allows the monitoring neuronal pathology and may provide diagnosis and prognosis in humans.

We are interested in pNF-H as biomarker of neuronal injury in spinal cord injury and we used a pNF-H ELISA test capable of detecting the levels of phosphorylated NF-H (pNF-H) to patients with spinal cord injury. We studied the pNF-H levels in CSF in two patients with spinal cord injury (SCI) and for normal values of $\mathrm{pNF}-\mathrm{H}$ we determined the CSF pNF-H level from individuals without neurological damage.

The pNF-H values of CSF from the two patients with SCI were 5-10 times higher than the normal and its higher values were related to an unfavorable outcome.

In conclusion, although the number of cases is very low - only two, in the context of experimental studies in animals with SCI, we can say that pNF-H is marker in SCI in humans and its increased values are consistent with an unfavorable outcome.

Key words: biomarker, phosphorylated neurofilament subunit, spinal cord injury.

\section{Introduction}

Three major subunits of neurofilaments are namely heavy (NF-H), medium (NF$\mathrm{M})$ and light (NF-L) subunits on the basis of molecular weight and these neurofilament are concentrated in large motor axons. The phosphorylated neurofilament subunit NF-H (pNF-H) is present into CSF in significant amounts following neuronal injury and may be detected. Several studies showed the pNF$\mathrm{H}$ is a biomarker of the neuronal injuries and its detection allows the monitoring neuronal pathology and we wished to study the pNF-H levels in CSF as biomarker of neuronal injury in spinal cord injury.

We measured pNF-H concentration by ELISA test in CSF in two spinal cord injury patients and and we correlated the values of $\mathrm{pNF}-\mathrm{H}$ with the clinical evolution, also we measured the normal 
values in samples obtained by lumbar puncture from individuals without neurologic disorders.

\section{Cases presentation}

\section{Case 1}

A 50-years old man with acute cervical spine injury and tetraplegia after a motor vehicle accident was admited 3 hours after the accident. Radiology and cervical CT showed a bilateral C5-C6 facet dislocation and anterior luxation. (figure 1) The patient had marked vegetative disorders (neurogenic shock, respiratory failure, priapism etc.) and the treatment in intensive care unit included high doses of methylprednisolone and cervical spinal traction to reduce the luxation. Unfortunately evolution was unfavorable with death in 3 days.

\section{Case 2}

A 53-years old man with acute cervical spine spine injury with tetraplegia after fall from a horse cart was admited 5 hours after the accident. Radiology and cervical CT showed a unilateral C4-C5 dislocation with subluxation and spinal cord compresion. Immediate surgical treatment

consisted of anterior cervical approach with reduction and decompresion and stabilsation with autologous iliac crest bone graft. Favorable evolution with CSF samples obtained before surgery and each day for four days postoperatively.

\section{Results}

We determined the normal level of pNF-H in samples obtained by lumbar puncture from five individuals without neurologic disorders and its values are similar to the normal level set by Petzold and Shaw in 2007: $0 \mathrm{ng} / \mathrm{mL}$ to $0.9 \mathrm{ng} / \mathrm{mL}$.

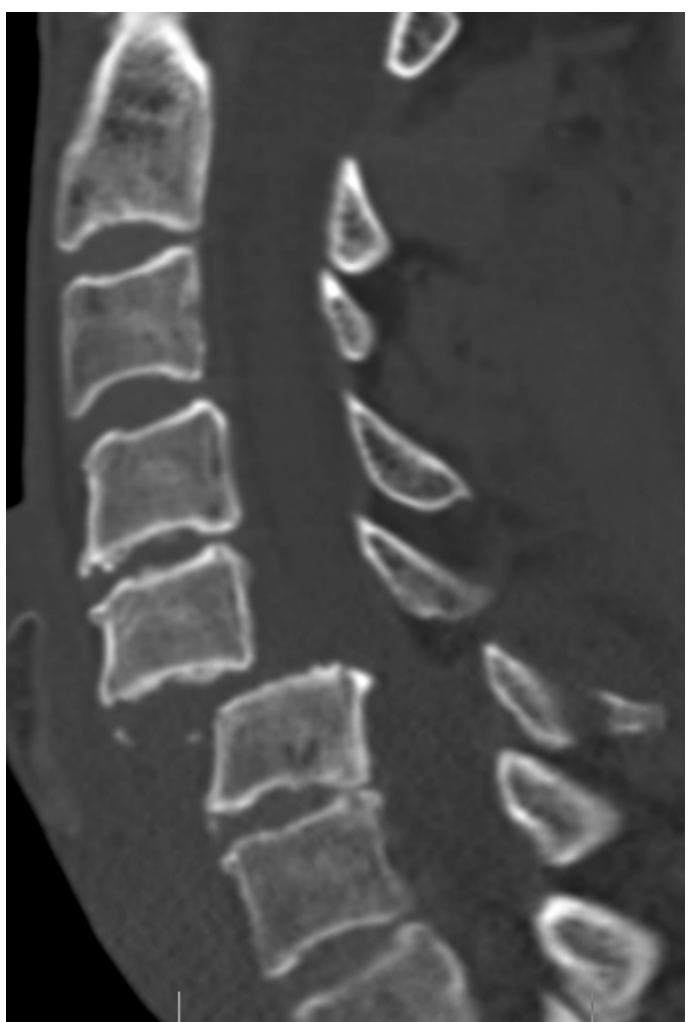

Figure 1 Case 1 - C5 - C6 luxation and tetraplegia

TABLE 1

Values of pNF-H in CSF (ng/mL)

\begin{tabular}{|c|c|c|c|c|c|}
\hline & Day 1 & Day 2 & Day 3 & Day 4 & Day 5 \\
\hline Case 1 & 9.3 & 10.9 & 9.3 & & \\
\hline Case 2 & 4.5 & 5.5 & 5.9 & 6.5 & 5.6 \\
\hline Normal 1 & 0.2 & 0.2 & & & \\
\hline Normal 2 & 0 & & & & \\
\hline Normal 3 & 0 & & & & \\
\hline Normal 4 & 0.9 & 0.9 & & & \\
\hline Normal 5 & 0.1 & 0.1 & & & \\
\hline
\end{tabular}

The normal values and the values of pNF-H in the two cases are shown in the table 1.

The normal level of pNF-H in human CSF was set less than or equal to 0.94 $\mathrm{ng} / \mathrm{mL}$ (Petzold and Shaw, 2007).

\section{Discussion and conclusion}

There are only two cases of spinal cord injury with CSF phosphorylated 
neurofilament subunit NF-H (pNF-H) levels measurement. The normal values of pNF-H from five individuals without neurologic disorders are similar to the normal level set by Petzold and Shaw in 2007: less than or equal to $0.94 \mathrm{ng} / \mathrm{mL}$.

The pNF-H values of CSF from the two patients with SCI were 5-10 times higher than the normal. The case 1 of spinal cord injury with severe evolution and death had ten times higher values of pNF-H than the normal and the case 2 with favorable evolution had only five or six times higher of pNF-H values than the normal.

There are several studies on the phosphorylated form of the neurofilament subunit NF-H (pNF-H) related to neuronal injuries but few data on pathological values of pNF-H in CSF in patients with spinal cord injury. Although there are only two cases, the results are similar to the experimental data, but it need analysis of a statistically significant number of cases in order to confirm the value of biomarker pNF-H in spinal cord injury.

In conclusion the phosphorylated form of the neurofilament subunit NF-H (pNF$\mathrm{H}$ ) is biomarker in SCI in humans and its increased values are consistent with an unfavorable outcome.

This study about acute traumatic spinal cord injury is the subject of the grant:

"Immediate neuroprotective therapy in acute traumatic spinal cord injury", that won the 2011 national competition of National Research Council (CNCS), Ideas, grant number: PN-II-ID-PCE-2011-30569, funded by CNCS - UEFISCDI Romania.
Corresponding author:

A.St. Iencean

Neurosurgery, "Prof. Dr. Nicolae Oblu"

Hospital Iasi, andrei_steffan@yahoo.com

\section{References}

1. Gerry Shaw, Cui Yang, Rebecca Ellis, Kevin Anderson, J. Parker Mickle et all. Hyperphosphorylated neurofilament NF-H is a serum biomarker of axonal injury Biochemical and Biophysical Research Communications 336 (2005) 1268-1277

2. Axel Petzold, Gerry Shaw Comparison of two ELISA methods for measuring levels of the phosphorylated neurofilament heavy chain $\mathrm{J}$ of Immunological Methods 319 (2007) 34-40

3. Şt.M. Iencean, I. Poeată, Gh. Solcan, Dana Turliuc,E.C. Popescu, B. Costăchescu, A.Şt. Iencean: News and views of neuroprotection in complete traumatic spinal cord injuries, Romanian Neurosurgery, 2011, vol XVIII,4;412 - 420

4. St.M. Iencean, I. Poeata, Didona Ungureanu, D. Cuciureanu,B. Costachescu, Al. Chiriac. Traumatic spinal cord injuries: neuroprotection and recent outcomes Romanian Neurosurgery, 2012, vol XIX,3; 210- 216

5. D de Jong, R W M M Jansen, Y A L Pijnenburg, W J A van Geel, G F Borm, H P H Kremer, M M Verbeek: CSF neurofilament proteins in the differential diagnosis of dementia J Neurol Neurosurg Psychiatry 2007;78:936-938

6. Johannes Brettschneider, Axel Petzold, Sigurd D. Sußmuth, G B. Landwehrmeyer et all. Neurofilament Heavy-Chain NfH in Cerebrospinal Fluid Supports the Differential Diagnosis of Parkinsonian Syndromes Movement Disorders, Vol. 21, No. 12, 2006

7. Melissa M. Gresle, Helmut Butzkueven, and Gerry Shaw Neurofilament Proteins as Body Fluid Biomarkers of Neurodegeneration inMultiple Sclerosis Multiple Sclerosis International, Volume 2011, Article ID 315406, 7

8. Stephen B Lewis, Regina A Wolper, Lynn Miralia, Cui Yang and Gerry Shaw Detection of phosphorylated NF-H in the cerebrospinal fluid and blood of aneurysmal subarachnoid hemorrhage patients $\mathrm{J}$ of Cerebral Blood Flow \& Metabolism (2008) 28, 1261

9. Kevin J. Anderson, Stephen W. Scheff, Kelly M. Miller, Kelly N. Roberts, et all. The Phosphorylated Axonal Form of the Neurofilament Subunit NF-H (pNF-H) as a Blood Biomarker of Traumatic Brain Injury J of Neurotrauma, 2008, $25: 1079-1085$

10. Petzold A, Keir G, Warren J, Fox N, Rossor MN. A systematic review and meta-analysis of CSF neurofilament protein levels as biomarkers in dementia. Neurodegener Dis. 2007;4(2-3):185-94. 